\title{
The Classification of Traveling Wave Solutions to Space-Time Fractional Resonance Nonlinear Schrödinger Type Equation with a Special Kind of Fractional Derivative
}

\author{
Lianguang Jia and Li Tang $\mathbb{D}$ \\ Teacher Education Institute, Daqing Normal University, Daqing, 163712 Heilongjiang, China \\ Correspondence should be addressed to Li Tang; tang2098@126.com
}

Received 6 April 2021; Revised 16 June 2021; Accepted 28 June 2021; Published 12 July 2021

Academic Editor: Maria L. Gandarias

Copyright (C) 2021 Lianguang Jia and Li Tang. This is an open access article distributed under the Creative Commons Attribution License, which permits unrestricted use, distribution, and reproduction in any medium, provided the original work is properly cited.

\begin{abstract}
In this paper, the classification of single traveling wave solutions to a special kind of space-time fractional Schrödinger type equation with high-order nonlinearities is obtained by the complete discrimination system for polynomial method, where the fractional derivative is defined by Atangana's conformable definition. The descriptions of the method and the fractional derivative are given, and the concrete procedure of the method is also presented in the paper. Especially, some new solutions such as hyperelliptic functions solution could be found and all the existing results about traveling wave solutions to the equation could also be seen. Moreover, concrete examples indicate that all the solutions obtained in the paper can be realized.
\end{abstract}

\section{Introduction}

In order to portray anomalous phenomena in science and engineering, nonlinear equation with fractional order derivative is proposed and has been widely applied in the fields such as anomalous diffusion $[1,2]$, and thus, finding exact solutions to such a special kind of equation is an important and significant task. And fractional derivative also has many definitions, for example, Caputo derivative [3] and RiemannLiouville derivative [4]. However, some of the definitions are very complicated that it is too difficult to find expected exact solutions to the corresponding equation and some definitions such as modified Riemann-Liouville derivative have already been proved wrong $[5,6]$. Recently, Atangana et al. proposed a special kind of definition called Atangana's fractional derivative [7], which is easy to apply due to the reason that it obeys almost all the properties satisfied by the conventional Newtonian concept of derivative such as chain rules.
The definition of the derivative is given as follows:

$$
{ }_{0}^{A} D_{t}^{\alpha}(\phi(t))=\lim _{\mathscr{E} \longrightarrow 0} \frac{\phi\left[t+\mathscr{E}(t+(1 / \Gamma(\alpha)))^{1-\alpha}\right]-\phi(t)}{\mathscr{E}} .
$$

If the above limitation holds, then we would have the following properties [7].

$$
\begin{gathered}
(1)_{0}^{A} D_{t}^{\alpha}(\phi(t) \pm \lambda(t))={ }_{0}^{A} D_{t}^{\alpha}(\phi(t)) \pm{ }_{0}^{A} D_{t}^{\alpha}(\lambda(t)), \\
(2){ }_{0}^{A} D_{t}^{\alpha}(\phi(t) \lambda(t))=\lambda(t){ }_{0}^{A} D_{t}^{\alpha}(\phi(t))+\phi(t){ }_{0}^{A} D_{0}^{A}(\lambda(t)), \\
(3){ }_{0}^{A} D_{t}^{\alpha}\left(\frac{\phi(t)}{\lambda(t)}\right)=\frac{\lambda(t){ }_{0}^{A} D_{t}^{\alpha}(\phi(t))-\phi(t){ }_{0}^{A} D_{t}^{\alpha}(\lambda(t))}{\lambda^{2}(t)}, \\
(4){ }_{0}^{A} D_{t}^{\alpha}(c)=0,
\end{gathered}
$$


where $\phi(t)$ and $\lambda(t)$ are the two $\beta$-differentiable functions with $\beta \in(0,1]$. Via this definition, many interesting and important results have been proposed [8-10].

In this paper, we consider the following space-time fractional resonance nonlinear Schrödinger type equation with Atangana's derivative [11].

$$
\begin{aligned}
i_{0}^{A} D_{t}^{\alpha} u(z, t)_{0}^{A} D_{t}^{\alpha} & +\rho_{0}^{A} D_{0}^{2 \alpha} u(z, t)+\left(\alpha|u|+\beta\left|u^{2}\right|+\gamma|u|^{3}\right) u(z, t) \\
& +r \frac{{ }_{0}^{A} D_{z}^{2 \alpha}\left(\left|u^{2}\right|\right)}{|u|} u(z, t)=0,
\end{aligned}
$$

where $z$ is the longitudinal coordinate along the optical fibers, $u(z, t)$ represents the normalized electric-field envelope, ${ }_{0}^{A} D_{t}^{\alpha}$ and ${ }_{0}^{A} D_{z}^{2 \alpha}$ are the Atangana's fractional derivative with respect to $t$ and $z, r$ is the coefficient of resonant nonlinearity, and $\alpha, \beta$, and $\gamma$ are the corresponding coefficients of highorder nonlinearities. This equation is widely used in fluid dynamics, especially the Madelung's fluids in several nonlinear systems [12-14]. And in other fields such as nonlinear optics $[15,16]$ and plasma and nuclear physics [17-20], the resonance nonlinear Schrödinger equation also plays a vital role. In [11], Yepezmartinez and Gomezaguilar obtained soliton solutions to some forms of deformation of Equation (3) by the subequation method. His results are interesting.

Nowadays, many famous and important methods are proposed to achieve the goal of finding the exact solutions to the nonlinear fractional PDEs due to the significance of them, for example, subequation method [21, 22], $\left(G^{\prime} / G\right)$ expansion method [23, 24], and Sine-Gordon equation expansion method $[25,26]$. Other results about exact solutions to nonlinear partial differential equations can be seen in [27-31]. However, though the mentioned methods are very powerful, they cannot find all the exact traveling wave solutions to the expected equation, and some kinds of solutions such as elliptic function double periodic solutions could hardly be found. In order to overcome these difficulties, Liu proposed a powerful method called complete discrimination system for polynomial method [32-35]. This method could not only be used to solve integer-order differential equations [36-38] but can also get exact solutions to nonlinear fractional-order equations [39-44]. Moreover, besides obtaining the exact solutions, Kai et al. also find that this method could be used to get qualitative properties to nonlinear equations $[45,46]$. So, in this paper, we firstly transform Equation (3) into an ordinary differential equation by a special kind of fractional complex traveling wave transformation and then use the complete discrimination system for polynomial method of 5 th order to get the classification of the traveling wave solutions to it. Some new solutions such as hyperelliptic function solutions are given, and concrete examples with concrete parameters are also presented to ensure the existence of each solution obtained in the paper. This indicates that the complete discrimination system for polynomial method is efficient and powerful in handing the nonlinear problems arising in mathematics and physics.
The construction of the present paper is as follows. We first give a brief introduction to the complete discrimination system for polynomial method in Section 2, and then transform the original equation into the ordinary differential equation and solve it in Section 3. Moreover, concrete examples are also presented in this section. In the final, a conclusion is drawn in Section 4.

\section{Complete Discrimination System for Polynomial Method}

The main idea of the complete discrimination system for polynomial method can be concluded as follows. When we encounter with a nonlinear differential equation

$$
F\left(u, u_{t}, u_{z}, u_{t t}, \cdots\right)=0
$$

then we need to take a traveling wave transformation

$$
\xi=k x+\omega t
$$

to transform it into

$$
F\left(u, u^{\prime}, u^{\prime \prime}, \cdots\right)=0
$$

If Equation (6) could be written into the following integral form,

$$
\xi-\xi_{0}=\int \frac{d u}{\sqrt{G(u)}},
$$

where $G(u)$ could be any type of function such as rational function. Then, we can just obtain the classification of single traveling wave solutions to the original equation by discussing the relationship between the roots and the parameters of $G(u)$. We can see it clearly that the steps of using complete discrimination system for polynomial method can be given as follows:

Step 1. Take the traveling wave transformation to transform the original equation into the ordinary equation.

Step 2. Rewrite the equation into the integral form.

Step 3. Obtain the exact solutions to the original equation by discussing the relationship between the roots and the coefficients.

In the next section, we will use this method to obtain the classification of traveling wave solutions to Equation (3).

\section{The Classification of the Traveling Wave Solutions to Equation (3)}

To solve Equation (3), we first take the following fractional complex transformation:

$$
u(z, t)=F(\xi) \exp (i \theta)
$$


where

$$
\begin{gathered}
\xi=\frac{k}{\alpha}\left[\left(z+\frac{1}{\Gamma(\alpha)}\right)^{\alpha}+v\left(t+\frac{1}{\Gamma(\alpha)}\right)^{\alpha}\right], \\
\theta=-\frac{\kappa}{\alpha}\left[\left(z+\frac{1}{\Gamma(\alpha)}\right)^{\alpha}+\omega\left(t+\frac{1}{\Gamma(\alpha)}\right)^{\alpha}\right],
\end{gathered}
$$

and $\theta, \kappa, \omega, v$, and $k$ are all real constants. Then by substituting Equation (9) into Equation (3), we can get the following:

$$
v=2 \kappa \rho,
$$

for the imaginary part and

$$
-\left(\kappa^{2} \rho+\omega\right) F+\alpha F^{2}+\beta F^{3}+\gamma F^{4}+k^{2}(r+\rho) F^{\prime \prime}=0,
$$

for the real part. Multiplying both sides with $F^{\prime}$ and integrating it once yields

$$
-\frac{\left(\kappa^{2} \rho+\omega\right)}{2} F^{2}+\frac{\alpha}{3} F^{3}+\frac{\beta}{4} F^{4}+\frac{\gamma}{5} F^{5}+\frac{k^{2}(r+\rho)}{2}\left(F^{\prime}\right)^{2}=c,
$$

then we have the following:

$$
\left(F^{\prime}\right)^{2}=a_{5} F^{5}+a_{4} F^{4}+a_{3} F^{3}+a_{2} F^{2}+a_{0}
$$

where $a_{5}=-2 \gamma / 5 k^{2}(r+\rho), a_{4}=-\beta / 2 k^{2}(r+\rho), a_{3}=-2 \alpha / 3 k^{3}$ $(r+\rho), a_{2}=-\left(k^{2} \rho+\omega\right) /\left(k^{2}(r+\rho)\right)$, and $a_{0}=2 c / k^{2}(r+\rho)$. Via the transformation

$$
\begin{aligned}
\varphi & =a_{5}^{1 / 5}\left(F+\frac{a_{4}}{5 a_{5}}\right), \\
\xi_{1} & =a_{5}^{1 / 5} \xi
\end{aligned}
$$

we can obtain the following equation:

$$
\left(\varphi^{\prime}\right)^{2}=\varphi^{5}+b_{3} \varphi^{2}+b_{2} \varphi^{2}+b_{1} \varphi+b_{0}=G(\varphi)
$$

where $b_{3}=-2 / 5 a_{4}^{2} a_{4}^{-5 / 8}+a_{3} a_{5}^{-3 / 5}, b_{2}=4 / 25 a_{4}^{3} a_{5}^{-12 / 5}+a_{2} a_{5}^{-2 / 5}$, $b_{1}=-3 / 125 a_{4}^{4} a_{5}^{-16 / 5}+a_{2} a_{5}^{-2 / 5}$, and $b_{0}=4 / 3125 a_{4}^{4} a_{5}^{-5}+1 / 25 a_{2}$ $a_{4}^{2} a_{5}^{-2}+a_{0}$. Thus, we can get the following:

$$
\pm\left(\xi_{1}-\xi_{0}\right)=\int \frac{d \varphi}{\sqrt{G(\varphi)}},
$$

and then the corresponding complete discrimination system is given as follows:

$$
\begin{aligned}
D_{2}= & -b_{3}, \\
D_{3}= & 40 b_{1} b_{3}-12 b_{3}^{3}-45 b_{2}^{2}, \\
D_{4}= & -4 b_{2}^{2} b_{3}^{3}+12 b_{1} b_{3}^{4}+117 b_{1} b_{2}^{2} b_{3}-88 b_{1}^{2} b_{3}^{2} \\
& -40 b_{0} b_{2} b_{3}^{2}-27 b_{1}^{3} b_{2}^{4}-300 b_{0} b_{1} b_{2}+160 b_{1}^{3},
\end{aligned}
$$

$$
\begin{aligned}
D_{5}= & -1600 b_{0} b_{2} b_{1}^{3}-3750 b_{2} b_{3} b_{0}^{3}+2000 b_{3} b_{0}^{2} b_{1}^{2} \\
& -4 b_{1}^{2} b_{2}^{2} b_{3}^{3}+16 b_{0} b_{2}^{3} b_{3}^{3}-900 b_{1} b_{0}^{2} b_{3}^{3}+825 b_{0}^{2} b_{2}^{2} b_{3}^{2} \\
& +144 b_{3} b_{2}^{2} b_{1}^{3}+2250 b_{1} b_{0}^{2} b_{2}^{2}+16 b_{1}^{3} b_{3}^{4}+108 b_{0}^{2} b_{3}^{5} \\
& -128 b_{1}^{4} b_{2}^{2}-27 b_{1}^{2} b_{2}^{4}+108 b_{0} b_{2}^{5}+256 b_{1}^{5}+3125 b_{0}^{4} \\
& -72 b 0 b_{1} b_{2} b_{3}^{4}+560 b_{0} b_{2} b_{1}^{2} b_{3}^{2}-630 b_{3} b_{1} b_{0} b_{2}^{3},
\end{aligned}
$$

$$
\begin{aligned}
E_{2}= & 160 b_{1}^{2} b_{3}^{3}+900 b_{1}^{2} b_{2}^{2}-48 b_{1} b_{3}^{5} \\
& +60 b_{1} b_{2}^{2} b_{3}^{2}+1500 b_{0} b_{1} b_{2} b_{3}+16 b_{2}^{2} b_{3}^{4} \\
& -1100 b_{0} b_{2} b_{3}^{3}+625 b_{0}^{2} b_{3}^{2}-3375 b_{0} b_{2}^{3},
\end{aligned}
$$

$$
F_{2}=3 b_{2}^{2}-8 b_{1} b_{3}
$$

Now we can apply the complete discrimination system for polynomial method to get the classification of single traveling wave solutions to the original equation, and twelve cases need to be discussed here. What has to be illustrated here is that the values of parameters are not from literature; they are just estimated to show the corresponding solutions directly.

Case 1. $D_{3}>0, D_{4}=0, D_{5}=0$, and $E_{2} \neq 0 . G(\varphi)$ is given as follows:

$$
G=(\varphi)=\left(\varphi-\beta_{1}\right)^{2}\left(\varphi-\beta_{2}\right)^{2}\left(\varphi-\beta_{3}\right),
$$

where $\beta_{1}, \beta_{2}$, and $\beta_{3}$ are the corresponding three roots satisfying $2 \beta_{1}+2 \beta_{2}+\beta_{3}=0$. Then, the solutions in different conditions to the original equation shall be shown as follows:

$$
\begin{aligned}
\pm \frac{\beta_{2}-\beta_{1}}{2}\left(\xi_{1}-\xi_{0}\right)= & \sqrt{\beta_{3}-\beta_{1}} \arctan \sqrt{\frac{\varphi-\beta_{3}}{\beta_{3}-\beta_{1}}} \\
& -\sqrt{\beta_{3}-\beta_{2}} \arctan \sqrt{\frac{\varphi-\beta_{3}}{\beta_{3}-\beta_{2}}} \\
& \cdot\left(\beta_{3}>\beta_{1}, \beta_{3}>\beta_{2}\right),
\end{aligned}
$$




$$
\begin{aligned}
\pm \frac{\beta_{2}-\beta_{1}}{2}\left(\xi_{1}-\xi_{0}\right)= & \sqrt{\beta_{3}-\beta_{1}} \arctan \sqrt{\frac{\varphi-\beta_{3}}{\beta_{3}-\beta_{1}}} \\
& -\frac{1}{\sqrt{\beta_{3}-\beta_{2}}} \ln \left|\frac{\sqrt{\varphi-\beta_{3}}-\sqrt{\beta_{2}-\beta_{3}}}{\sqrt{\varphi-\beta_{2}}+\sqrt{\beta_{2}-\beta_{3}}}\right| \\
& \cdot\left(\beta_{1}<\beta_{3}<\beta_{2}\right),
\end{aligned}
$$

$$
\begin{aligned}
\pm \frac{\beta_{2}-\beta_{1}}{2}\left(\xi_{1}-\xi_{0}\right)= & -\sqrt{\beta_{3}-\beta_{2}} \arctan \sqrt{\frac{\varphi-\beta_{3}}{\beta_{3}-\beta_{1}}} \\
& +\frac{1}{\sqrt{\beta_{1}-\beta_{3}}} \ln \left|\frac{\sqrt{\varphi-\beta_{3}}-\sqrt{\beta_{1}-\beta_{3}}}{\sqrt{\varphi-\beta_{3}}+\sqrt{\beta_{1}-\beta_{3}}}\right| \\
& \cdot\left(\beta_{2}<\beta_{3}<\beta_{1}\right),
\end{aligned}
$$

and

$$
\begin{aligned}
\pm \frac{\beta_{2}-\beta_{1}}{2}\left(\xi_{1}-\xi_{0}\right)= & \frac{1}{2 \sqrt{\beta_{1}-\beta_{3}}} \ln \left|\frac{\sqrt{\varphi-\beta_{3}}-\sqrt{\beta_{1}-\beta_{3}}}{\sqrt{\varphi-\beta_{3}}+\sqrt{\beta_{1}-\beta_{3}}}\right| \\
& -\frac{1}{2 \sqrt{\beta_{2}-\beta_{3}}} \ln \left|\frac{\sqrt{\varphi-\beta_{3}}-\sqrt{\beta_{2}-\beta_{3}}}{\sqrt{\varphi-\beta_{3}}+\sqrt{\beta_{2}-\beta_{3}}}\right| \\
& \cdot\left(\beta_{3}<\beta_{1}, \beta_{3}<\beta_{2}\right) .
\end{aligned}
$$

For example, when $k=1, \kappa=1, r=-2, \rho=1, \gamma=5, \alpha=-9$ , $\beta=0, \omega=-5$, and $c=0$, namely, $b_{3}=-3, b_{2}=2$, and $b_{1}=$ $b_{0}=0$, then we have $\beta_{1}=0, \beta_{2}=1$, and $\beta_{3}=-2$ and the solution is as follows:

$\pm\left(\xi-\xi_{0}\right)=\frac{1}{2 \sqrt{2}} \ln \left|\frac{\sqrt{\varphi+2}-\sqrt{2}}{\sqrt{\varphi+2}+\sqrt{2}}\right|-\frac{1}{2 \sqrt{3}} \ln \left|\frac{\sqrt{\varphi+2}-\sqrt{3}}{\sqrt{\varphi+2}+\sqrt{3}}\right|$.

Case 2. $D_{2} \neq 0, D_{3}=0, D_{4}=0, D_{5}=0$, and $F_{2} \neq 0$. Then,

$$
G(\varphi)=\left(\varphi-\beta_{1}\right)^{3}\left(\varphi-\beta_{2}\right)^{2}
$$

where $\beta_{1}$ and $\beta_{2}$ satisfy the relation $3 \beta_{1}+2 \beta_{2}=0$. We have the following:

$$
\begin{aligned}
\pm \frac{\beta_{2}-\beta_{1}}{2}\left(\xi_{1}-\xi_{0}\right)= & -\frac{1}{\sqrt{\varphi-\beta_{1}}}-\sqrt{\beta_{1}-\beta_{2}} \arctan \\
& \cdot \sqrt{\frac{\varphi-\beta_{1}}{\beta_{1}-\beta_{2}}}\left(\beta_{1}>\beta_{2}\right),
\end{aligned}
$$

$$
\begin{aligned}
\pm \frac{\beta_{2}-\beta_{1}}{2}\left(\xi_{1}-\xi_{0}\right)= & -\frac{1}{\sqrt{\varphi-\beta_{1}}}-\frac{1}{2 \sqrt{\beta_{2}-\beta_{1}}} \ln \\
& \cdot\left|\frac{\sqrt{\varphi-\beta_{1}}-\sqrt{\beta_{2}-\beta_{1}}}{\sqrt{\varphi-\beta_{1}}+\sqrt{\beta_{1}-\beta_{3}}}\right|\left(\beta_{1}<\beta_{2}\right) .
\end{aligned}
$$

For instance, when $k=1, \kappa=10, r=-2, \rho=1, \gamma=5, \alpha=$ $-15, \beta=-20, \omega=-10$, and $c=108$, namely, $b_{3}=-15, b_{2}=$ $10, b_{1}=60$, and $b_{0}=-72$, then we have $\beta_{1}=2, \beta_{2}=-3$, and the following solution:

$$
\pm \frac{5}{2}\left(\xi-\xi_{0}\right)=-\frac{1}{\sqrt{\varphi-2}}-\sqrt{5} \arctan \sqrt{\frac{\varphi-2}{5}} .
$$

Case 3. When $D_{2} \neq 0, D_{3}=0, D_{4}=0, D_{5}=0$, and $F_{2}=0$, we have the following:

$$
F(\varphi)=\left(\varphi-\beta_{1}\right)^{4}\left(\varphi-\beta_{2}\right),
$$

where $\beta_{1}$ and $\beta_{2}$ are the two different real constants satisfying $4 \beta_{1}+\beta_{2}=0$. Then, the solution of Equation (3) can be shown as follows:

$$
\begin{aligned}
\pm \frac{\beta_{2}-\beta_{1}}{2}\left(\xi_{1}-\xi_{0}\right)= & -\frac{\sqrt{\varphi-\beta_{2}}}{2\left(\varphi-\beta_{1}\right)}-\frac{1}{4 \sqrt{\beta_{1}-\beta_{2}}} \ln \\
& \cdot\left|\frac{\sqrt{\varphi-\beta_{2}}-\sqrt{\beta_{1}-\beta_{2}}}{\sqrt{\varphi-\beta_{2}}+\sqrt{\beta_{1}-\beta_{2}}}\right|\left(\beta_{1}>\beta_{2}\right),
\end{aligned}
$$

$$
\begin{aligned}
\pm \frac{\beta_{2}-\beta_{1}}{2}\left(\xi_{1}-\xi_{0}\right)= & -\frac{\sqrt{\varphi-\beta_{2}}}{2\left(\varphi-\beta_{1}\right)}-\frac{1}{2 \sqrt{\beta_{2}-\beta_{1}}} \arctan \\
& \cdot \sqrt{\frac{\varphi-\beta_{2}}{\beta_{2}-\beta_{1}}}\left(\beta_{1}<\beta_{2}\right) .
\end{aligned}
$$

Take the concrete parameters $k=1, \kappa=0, r=-2, \rho=1$, $\gamma=5, \alpha=0, \beta=20, \omega=0$, and $c=0$ as an illustrative example, which yields $b_{3}=-10, b=20, b_{1}=-15$, and $b_{0}=4$. Thus, when $\beta_{1}=1$ and $\beta_{2}=-4$, then we can get the following:

$$
\pm \frac{5}{2}=\left(\xi-\xi_{0}\right)=-\frac{\sqrt{\varphi+4}}{2 \sqrt{\varphi-1}}-\frac{1}{2 \sqrt{5}} \ln \left|\frac{\sqrt{\varphi+4}-\sqrt{5}}{\sqrt{\varphi+4}+\sqrt{5}}\right| .
$$

Case 4. $D_{2}=D_{3}=D_{4}=D_{5}=0$. Then,

$$
F(\varphi)=\varphi^{5} .
$$

This leads to the following:

$$
\pm\left(\xi_{1}-\xi_{0}\right)=-\frac{2}{3} \varphi^{-3 / 2}
$$


which yields

$$
\varphi=\left[\frac{3}{2}\left(\xi_{1}-\xi_{0}\right)\right]^{2 / 3}
$$

For example, when $k=0, r=-2, \rho=1$, and $\kappa=\gamma=\alpha=\beta$ $=\omega=c=0$, then the corresponding solution is given by Equation (34).

Case 5. $D_{3}<0, D_{4}=0, D_{5}=0$, and $E_{2}=0 . G(\varphi)$ is given as follows:

$$
G(\varphi)=\left(\varphi-\beta_{1}\right)\left(\varphi^{2}+\beta_{2} \varphi+\beta_{3}\right)^{2}
$$

where $\beta_{1}, \beta_{2}$, and $\beta_{3}$ are the three different roots satisfying $\beta_{2}^{2}-4 \beta_{3}<0$ and $2 \beta_{2}=\beta_{1}$; thus, we have the following:

$$
\begin{aligned}
\pm\left(\xi_{1}+\xi_{0}\right)= & -\frac{2}{\rho \sqrt{4 \beta_{3}-\beta_{2}^{2}}}\left(\cos \delta \arctan \frac{2 \rho \sin \delta \sqrt{\varphi-\beta_{1}}}{\varphi-\beta_{1}-\rho^{2}}\right. \\
& \left.+\frac{\sin \delta}{2} \ln \frac{\varphi-\beta_{1}-\rho^{2}-2 \rho \cos \delta \sqrt{\varphi-\beta_{1}}}{\varphi-\beta_{1}-\rho^{2}+2 \rho \cos \delta \sqrt{\varphi-\beta_{1}}}\right),
\end{aligned}
$$

where $\quad \rho=\left(\beta_{1}^{2}+\beta_{1} \beta_{2}+\beta_{3}\right)^{1 / 4}$ and $\delta=-1 / 2 \arctan$ $\sqrt{4 \beta_{3}-\beta_{2}^{2}} / 2 \beta_{1}+\beta_{2}$.

Take the concrete parameters $k=1, \kappa=1, r=-2, \rho=1$, $\gamma=5, \alpha=87 / 16, \beta=0, \omega=21 / 8$, and $c=-1 / 8$ as an illustrative example; then, $b_{3}=-29 / 16, b_{2}=-17 / 32, b_{1}=0$, and $b_{0}$ $=-1 / 8$; thus, $\beta_{1}=1 / 2$ and $\beta_{2}=\beta_{3}=1 / 4$; then, we can get the following:

$$
\begin{aligned}
\pm\left(\xi-\xi_{0}\right)= & -\frac{8}{\rho \sqrt{17}}\left(\cos \delta \arctan \frac{2 \rho \sin \delta \sqrt{\varphi-1 / 2}}{\varphi-1 / 2-\rho^{2}}\right. \\
& \left.+\frac{\sin \delta}{2} \ln \frac{\varphi-1 / 2-\rho^{2}-2 \rho \cos \delta \sqrt{\varphi-1 / 2}}{\varphi-1 / 2-\rho^{2}+2 \rho \cos \delta \sqrt{\varphi-1 / 2}}\right)
\end{aligned}
$$

where $\rho=(5 / 8)^{1 / 4}$ and $\delta=-1 / 2 \arctan \sqrt{17} / 5$.

Case 6. When $D_{4}>0$ and $D_{5}=0$, we have the following:

$$
G(\varphi)=\left(\varphi-\beta_{1}\right)^{2}\left(\varphi-\beta_{2}\right)\left(\varphi-\beta_{3}\right)\left(\varphi-\beta_{4}\right)
$$

where $\beta_{i}(i=1,2,3,4)$ are the four distinct real roots satisfying $2 \beta_{1}+\beta_{2}+\beta_{3}+\beta_{4}=0$. Thus, we can get the following:

$$
\begin{aligned}
\pm\left(\xi_{1}-\xi_{0}\right)= & -\frac{2}{\left(\beta_{1}-\beta_{3}\right) \sqrt{\beta_{3}-\beta_{4}}} \\
& \cdot\left\{F(\eta, l)-\frac{\beta_{2}-\beta_{3}}{\beta_{2}-\beta_{1}} \prod\left(\eta, \frac{\beta_{2}-\beta_{3}}{\beta_{2}-\beta_{1}}, l\right)\right\},
\end{aligned}
$$

where

$$
\begin{gathered}
F(\eta, l)=\int_{0}^{\eta} \frac{d \eta}{\sqrt{1-l^{2} \sin ^{2} \eta}}, \\
\prod(\eta, n, l)=\int_{0}^{\eta} \frac{d \eta}{\left(1+n \sin ^{2} \eta\right) \sqrt{1-l^{2} \sin ^{2} \eta}} .
\end{gathered}
$$

For instance, when $k=1, \kappa=-4, r=0, \rho=-2, \gamma=5, \alpha$ $=-21, \beta=0, \omega=4$, and $c=0$, we have $b_{3}=-7, b_{2}=-6$, and $b_{1}=b_{0}=0$; thus, $\beta_{1}=0, \beta_{2}=1, \beta_{3}=2$, and $\beta_{4}=-3$; then, we can attain the solution to the original equation as follows:

$$
\pm\left(\xi-\xi_{0}\right)=\int \frac{d \varphi}{\sqrt{\varphi^{2}(\varphi-1)(\varphi-2)(\varphi+3)}}
$$

The explicit form of the solution could not be represented by any common function including the Jacobian elliptic function, so we only are given its integral expression here.

Case 7. $D_{3}<0$ and $D_{4}=D_{5}=E_{2}=0$. Then, we have the following:

$$
G(\varphi)=\left(\varphi-\beta_{1}\right)^{3}\left[\left(\varphi-\beta_{2}\right)^{2}+\beta_{3}^{2}\right]
$$

where $\beta_{1}, \beta_{2}$, and $\beta_{3}$ are the corresponding three roots satisfying $3 \beta_{1}+2 \beta_{2}=0$.

Thus, the solution of Equation (3) can be obtained as follows:

$$
\begin{aligned}
\pm\left(\xi_{1}-\xi_{0}\right)= & \frac{\tan \theta+\cot \theta}{2\left(\beta_{3} \tan \theta-\beta_{2}-\beta_{1}\right) \sqrt{\beta_{3} / \sin ^{3} 2 \theta}} F(\eta, s) \\
& -\frac{\beta_{3} \tan \theta+\beta_{3} \cot \theta}{\beta_{3} \cot \theta+\beta_{1}+\beta_{2}} \times\left\{\frac{\tan \theta+\beta_{1}+\beta_{2}}{\left(\beta_{3} \cot \theta+\beta_{2}-\beta_{1}\right) \sin \eta}\right. \\
& \left.\cdot \sqrt{ } 1-k^{2} \sin ^{2} \eta+F(\eta, l)-E(\eta, s)\right\}\left(\beta_{1} \neq \beta_{2}+\beta_{3}\right), \\
\pm\left(\xi_{1}-\xi_{0}\right)= & \sqrt{\frac{\sin ^{3} 2 \theta}{4 \beta_{3}^{3}}\left[\frac{1}{s} \arcsin (s \sin \eta)-F(\eta, s)\right]\left(\beta_{1}=\beta_{2}+\beta_{3}\right),}
\end{aligned}
$$

where $F(\eta, s)$ is defined by Equation (40), $\tan 2 \theta=\beta_{3} /\left(\beta_{1}-\right.$ $\left.\beta_{2}\right), s=\sin \theta(0<\theta<\pi / 2)$, and

$$
E(\eta, s)=\int_{0}^{\eta} \sqrt{1-s^{2} \sin ^{2} \zeta} d \zeta
$$

For instance, when $k=1, \kappa=0, r=0, \rho=-2, \gamma=0, \alpha=3$, $\beta=0, \omega=0$, and $c=0$, then $b_{3}=1$ and $b_{2}=b_{1}=b_{0}=0$, namely, $\beta_{1}=\beta_{2}=0$ and $\beta_{3}=1$; thus, we have the following:

$$
\pm\left(\xi-\xi_{0}\right)=\int \frac{d \varphi}{\varphi^{3 / 2} \sqrt{\varphi^{2}+1}} .
$$


Case 8. When $D_{4}<0$ and $D_{5}=0$, we have the following:

$$
G(\varphi)=\left(\varphi-\beta_{1}\right)^{2}\left(\varphi-\beta_{2}\right)\left[\left(\varphi-\beta_{3}\right)^{2}+\beta_{4}^{2}\right]
$$

where $\beta_{i}(i=1,2,3,4)$ are the real numbers satisfying $2 \beta_{1}+$ $2 \beta_{3}+\beta_{2}=0$. So, we have the following solutions:

$$
\begin{aligned}
\pm\left(\xi_{1}-\xi_{0}\right)= & \frac{\tan \theta+\cot \theta}{2\left(\beta_{3} \tan \theta-\beta_{2}-\beta_{1}\right) \sqrt{\beta_{3} / \sin ^{3} 2 \theta}} F(\eta, l) \\
& -\frac{\beta_{3} \tan \theta+\beta_{3} \cot \theta}{\beta_{3} \cot \theta+\beta_{1}+\beta_{2}} \times\left\{\frac{\tan \theta+\beta_{1}+\beta_{2}}{\left(\beta_{3} \cot \theta+\beta_{2}-\beta_{1}\right) \sin \eta}\right. \\
& \left.\cdot \sqrt{1-s^{2} \sin ^{2} \eta+F(\eta, l)-E(\eta, s)}\right\} \\
& \cdot\left(\beta_{1} \neq \beta_{3}-\beta_{4} \tan \theta, \beta_{1} \neq \beta_{3}+\beta_{4} \cot \theta\right),
\end{aligned}
$$

$$
\begin{aligned}
\pm\left(\xi_{1}-\xi_{0}\right)= & \sqrt{\frac{\sin ^{3} 2 \theta}{4 \beta_{4}^{3}}}\left[\frac{1}{2} \arcsin (l \sin \eta)-F(\eta, s)\right] \\
& \cdot\left(\beta_{1}=\beta_{3}-\beta_{4} \tan \theta\right), \\
\pm\left(\xi_{1}-\xi_{0}\right)= & \sqrt{\frac{\sin ^{3} 2 \theta}{4 \beta_{4}^{3}}\left[F(\eta, s)-\frac{1}{\sqrt{1-s^{2}}} \ln \right.} \\
& \left.\cdot \frac{\sqrt{1-s^{2} \sin ^{2} \eta}+\sqrt{1-s^{2}} \sin \eta}{\cos \eta}\right] \\
& \cdot\left(\beta_{1}=\beta_{3}+\beta_{4} \cot \theta\right),
\end{aligned}
$$

where $F(\eta, l)$ is also defined by $(40), \tan 2 \theta=\beta_{4} /\left(\beta_{2}-\beta_{1}\right), s$ $=\sin \theta(0<\theta<\pi / 2)$, and $E(\eta, s)$ is given by Equation (46).

For instance, when $k=1, \kappa=0, r=0, \rho=-2, \gamma=5, \alpha=0$, $\beta=0, \omega=8$, and $c=0$, then $b_{3}=0, b_{2}=-4$, and $b_{1}=b_{0}=0$, namely, $\beta_{1}=0, \beta_{2}=2, \beta_{3}=-1$, and $\beta_{4}=1$; thus, we can get the following solution:

$$
\pm\left(\xi-\xi_{0}\right)=\int \frac{d \varphi}{\varphi \sqrt{\varphi^{3}-4}}
$$

Case 9. $D_{3}>0$ and $D_{4}=D_{5}=E_{2}=0 . G(\varphi)$ is given as follows:

$$
G(\varphi)=\left(\varphi-\beta_{1}\right)^{3}\left(\varphi-\beta_{2}\right)\left(\varphi-\beta_{3}\right)
$$

where $\beta_{1}, \beta_{2}$, and $\beta_{3}$ satisfy the relation of $3 \beta_{1}+\beta_{2}+\beta_{3}=0$. Then, the solution shall be given as follows:

$$
\begin{aligned}
\pm\left(\xi_{1}-\xi_{0}\right)= & \frac{1}{\beta_{1}-\beta_{3}} E\left(\arcsin \sqrt{\frac{\beta_{1}-\beta_{3}}{\varphi-\beta_{3}}}, \frac{\beta_{2}-\beta_{3}}{\beta_{1}-\beta_{3}}\right) \\
& -\sqrt{\frac{\varphi-\beta_{2}}{\left(\varphi-\beta_{1}\right)\left(\varphi-\beta_{2}\right)}}
\end{aligned}
$$

where $E$ is defined by Equation (46).
For instance, when $k=1, \kappa=0, r=0, \rho=-2, \gamma=5, \alpha=-3$ , $\beta=0, \omega=0$, and $c=0$, then $b_{3}=-1$ and $b_{2}=b_{1}=b_{0}=0$, namely, $\beta_{1}=0, \beta_{2}=-1$, and $\beta_{3}=1$; thus, we have the following:

$$
\pm\left(\xi-\xi_{0}\right)=\int \frac{d \varphi}{\varphi^{3 / 2} \sqrt{\varphi^{2}-1}} .
$$

Case 10. $D_{2}, D_{3}, D_{4}, D_{5}>0 . F(\varphi)$ can be written as follows:

$$
F(\varphi)=\left(\varphi-\beta_{1}\right)\left(\varphi-\beta_{2}\right)\left(\varphi-\beta_{3}\right)\left(\varphi-\beta_{4}\right)\left(\varphi-\beta_{5}\right)
$$

where $\beta_{i}(i=1,2, \cdots, 5)$ are the different real constants satisfying $\sum_{i=1}^{5} \beta_{i}=0$. Then, the solution in hyperelliptic function form is given as follows:

$$
\pm\left(\xi_{1}-\xi_{0}\right)=\frac{d \varphi}{\sqrt{\left(\varphi-\beta_{1}\right)\left(\varphi-\beta_{2}\right)\left(\varphi-\beta_{3}\right)\left(\varphi-\beta_{4}\right)\left(\varphi-\beta_{5}\right)}}
$$

For instance, when $k=1, \kappa=1, r=-2, \rho=1, \gamma=5, \alpha=-3$ , $\beta=-76 / 5, \omega=-2$, and $c=-36 / 5$, then $\beta_{1}=6 / 25 \beta_{2}=31 / 25$ $\beta_{3}=-44 / 25 \beta_{4}=-49 / 25$, and $\beta_{5}=56 / 25$; thus, we have the following:

$$
\pm\left(\xi-\xi_{0}\right)=\int \frac{3125 d \varphi}{\sqrt{(\varphi-6)(\varphi-31)(\varphi+44)(\varphi+49)(\varphi-56)}} .
$$

Case 11. When $D_{2}, D_{3}, D_{4}, D_{5}>0$, we have the following:

$$
F(\varphi)=\left(\varphi-\beta_{1}\right)\left(\varphi-\beta_{2}\right)\left(\varphi-\beta_{3}\right)\left[\left(\varphi-\beta_{4}\right)^{2}+\beta_{5}^{2}\right]
$$

where $\beta_{i}(i=1,2, \cdots, 5)$ are the constants satisfying $\beta_{1}+\beta_{2}$ $+\beta_{3}+2 \beta_{4}=0$ and $\beta_{1} \neq \beta_{2} \neq \beta_{3}$. Then, the hyperelliptic function type solution is given as follows:

$$
\pm\left(\xi_{1}-\xi_{0}\right)=\frac{d \varphi}{\sqrt{\left(\varphi-\beta_{1}\right)\left(\varphi-\beta_{2}\right)\left(\varphi-\beta_{3}\right)\left[\left(\varphi-\beta_{4}\right)^{2}+\beta_{5}^{2}\right]}} .
$$

For instance, when $k=1, \kappa=1, r=-2, \rho=1, \gamma=5, \alpha=-3$ , $\beta=-76 / 5, \omega=-2$, and $c=-36 / 5$, then $\beta_{1}=8 / 15, \beta_{2}=23 / 15$ , $\beta_{3}=-17 / 15, \beta_{4}=-7 / 15$, and $\beta_{5}=1$, so the solution to the original equation is given as follows:

$$
\pm\left(\xi=\xi_{0}\right)=\int \frac{d \varphi}{\sqrt{\varphi^{5}-7 \varphi^{4} / 3+\varphi^{3}-\varphi^{2}+4 / 3}} .
$$

Case 12. $D_{5}>0 \wedge\left(D_{4} \leq 0 \vee D_{3} \leq 0 \vee D_{2} \leq 0\right)$, where $\wedge$ means "and" and $\vee$ means "or". We have the following:

$$
G(\varphi)=\left(\varphi-\beta_{1}\right)\left[\left(\varphi-\beta_{2}\right)^{2}+\beta_{3}^{2}\right]\left[\left(\varphi-\beta_{4}\right)^{2}+\beta_{5}^{2}\right]
$$




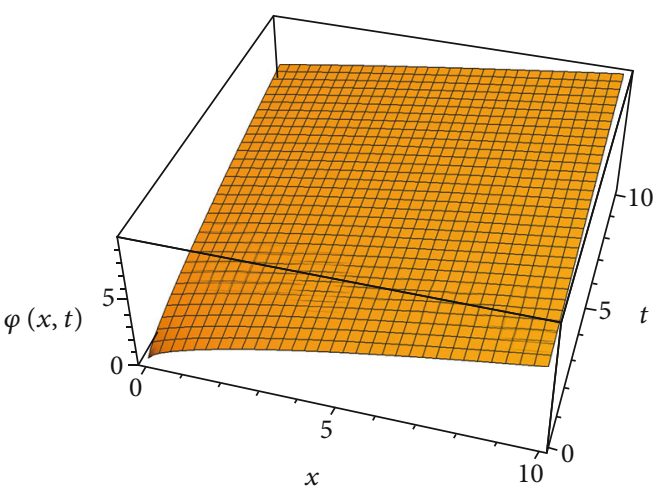

(a)

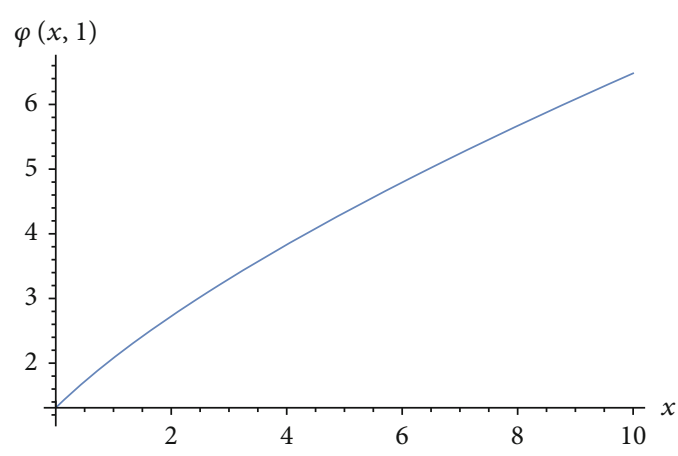

(b)

Figure 1: (a) The 3D graph of rational function solutions (Equation (34)) when $\alpha=0.5$. (b) The corresponding 2D plot.

where $\beta_{i}(i=1,2, \cdots, 5)$ are the roots satisfying $\beta_{1}+2 \beta_{2}+2$ $\beta_{4}=0$. Then, we can get the following hyperelliptic functions solution:

$$
\pm\left(\xi_{1}-\xi_{0}\right)=\int \frac{d \varphi}{\sqrt{\left(\varphi-\beta_{1}\right)\left[\left(\varphi-\beta_{2}\right)^{2}+\beta_{3}^{2}\right]\left[\left(\varphi-\beta_{4}\right)^{2}+\beta_{5}^{2}\right]}} .
$$

For instance, when $k=1, \kappa=1, r=-2, \rho=1, \gamma=5, \alpha=3$, $\beta=-4, \omega=-3$, and $c=2$, then $\beta_{1}=-6 / 5, \beta_{2}=-1 / 5, \beta_{3}=1$, $\beta_{4}=-4 / 5$, and $\beta_{5}=1$, so we have the following solution:

$\pm\left(\xi-\xi_{0}\right)=\int \frac{d \varphi}{\sqrt{\varphi^{5}+3 \varphi^{3} / 5}+36 \varphi^{2} / 25+62 \varphi / 125+6396 / 3125}$

From the results above, we can see that all the single traveling wave solutions, namely, the classification of single traveling wave solutions, are obtained. Besides the implicit solutions, Equation (34) is the rational function solution, and Equations (57), (61), and (64) are the hyperelliptic function solutions. Some of the results are new (i.e., Equation (61)), which shows the novelty of the present paper. However, we only show the single traveling wave solutions to Equation (3); other kinds of solutions such as multisoliton solution need to be further studied. Moreover, qualitative analysis to this equation should also be a future direction.

\section{Graphical Representations}

In this section, we give graphical representations to the solutions above. Since almost all solutions are implicit except Equation (34), and we really do not know how to present implicit function, thus we only show Equation (34) here.

From the figures above, we can see that the solutions given in the paper could be truly realized. From Figure 1, we can see that the amplitude increases with the time and the direction. Thus, the modulus becomes larger and larger with the process of evolution.

\section{Conclusion}

In this paper, the space-time fractional resonance nonlinear Schrödinger type equation with Atangana's derivative is considered by the complete discrimination system for polynomial method, and the present work would enrich the results of the fractional derivative, due to the reason that to the best of our knowledge, this is the first time that the hyperelliptic function solutions are obtained to the nonlinear fractional Schrödinger type equation. And this special kind of solution could hardly be obtained by other methods, which means new solutions are found. Moreover, the results presented in this paper show that the space-time fractional resonance nonlinear Schrödinger equation also has triangle function type periodic solutions and rational function type solutions. For each solution obtained in the paper, we have constructed concrete examples with concrete parameters so that it can be seen clearly that all the solutions given in the paper could be realized and the correctness of the results is also proved. Furthermore, considering the significance of the equation, our results would have practical applications in many scientific fields such as fluid dynamics.

\section{Data Availability}

Please contact authors for data requests.

\section{Conflicts of Interest}

The authors declare no conflicts of interest.

\section{Authors' Contributions}

Lianguang Jia and Li Tang have done all the work in the paper.

\section{Acknowledgments}

The authors would like to thank Chunyan Wang for her valuable help in improving the writing of the present paper. 


\section{References}

[1] B. Zheng, Y. Kai, W. Xu, N. Yang, K. Zhang, and P. M. Thibado, "Exact traveling and non-traveling wave solutions of the time fractional reaction-diffusion equation," Physica A: Statistical Mechanics and Its Applications, vol. 532, p. 121780, 2019.

[2] I. Podlubny, Fractional Differential Equations, Mathematics in Science and Engineering, Academic Press, San Diego, 1999.

[3] A. A. A. Kilbas, H. M. Srivastava, and J. J. Trujillo, "Theory and applications of fractional differential equations," North-Holland Mathematics Studies, vol. 204, 2006.

[4] M. Riesz, "L'int'egrale de Riemann-Liouville et le probl'eme de Cauchy," Acta Mathematica, vol. 81, no. 1, pp. 1-222, 1949.

[5] C. Liu, "Counterexamples on Jumarie's two basic fractional calculus formulae," Communications in Nonlinear Science and Numerical Simulation, vol. 22, no. 1-3, pp. 92-94, 2015.

[6] C. Liu, "Counterexamples on Jumarie's three basic fractional calculus formulae for non-differentiable continuous functions," Chaos, Solitons and Fractals, vol. 109, pp. 219-222, 2018.

[7] A. Atangana, D. Baleanu, and A. Alsaedi, "Analysis of timefractional Hunter-Saxton equation: a model of neumatic liquid crystal," Open Physics, vol. 14, no. 1, pp. 145-149, 2016.

[8] A. Yusuf, A. I. Aliyu, and D. Baleanu, "Conservation laws, soliton-like and stability analysis for the time fractional dispersive long-wave equation," Advances in Difference Equations, vol. 2018, Article ID 319, 2018.

[9] R. Weiguo, "Idea of invariant subspace combined with elementary integral method for investigating exact solutions of time-fractional NPDEs," Applied Mathematics and Computation, vol. 339, pp. 158-171, 2018.

[10] H. Yepezmartinez and J. F. Gomezaguilar, "Fractional subequation method for Hirota-Satsuma-coupled KdV equation and coupled $\mathrm{mKdV}$ equation using the Atangana's conformable derivative," Waves in Random and Complex Media, vol. 29, no. 4, pp. 678-693, 2019.

[11] H. Yepezmartinez and J. F. Gomezaguilar, "Optical solitons solution of resonance nonlinear Schrödinger type equation with Atangana's-conformable derivative using sub-equation method," Waves in Random and Complex Media, vol. 31, no. 3, pp. 573-596, 2021.

[12] H. Triki, A. Yildirim, T. Hayat, O. M. Aldossary, and A. Biswas, "1-Soliton solution of the generalized resonant nonlinear dispersive Schrödinger's equation with time dependent coefficients," Advanced Science Letters, vol. 16, no. 1, pp. 309-312, 2012.

[13] H. Triki, T. Hayat, O. M. Aldossary, and A. Biswas, "Bright and dark solitons for the resonant nonlinear Schrödinger's equation with time-dependent coefficients," Optics and Laser Technology, vol. 44, no. 7, pp. 2223-2231, 2012.

[14] M. Eslami, M. Mirzazadeh, and A. Biswas, "Soliton solutions of the resonant nonlinear Schrödinger's equation in optical fibers with time-dependent coefficients by simplest equation approach," Journal of Modern Optics, vol. 60, no. 19, pp. 1627-1636, 2013.

[15] C. Yan and Z. Yan, "New exact solutions of $(2+1)$-dimensional Gardner equation via the new Sine-Gordon equation expansion method," Physics Letters A, vol. 224, pp. 77-84, 1996.

[16] C. Yan, "A simple transformation for nonlinear waves," Physics Letters A, vol. 224, no. 1-2, pp. 77-84, 1996.
[17] G. P. Agrawal, Nonlinear Fiber Optics, Academic Press, New York, 1995.

[18] R. Guo and H. Q. Hao, "Breathers and localized solitons for the Hirota-Maxwell-Bloch system on constant backgrounds in erbium doped fibers," Annals of Physics, vol. 344, pp. 10-16, 2014.

[19] R. Guo, H. Q. Hao, and L. L. Zhang, "Dynamic behaviors of the breather solutions for the AB system in fluid mechanics," Nonlinear Dynamics, vol. 74, no. 3, p. 701709, 2013.

[20] Z. J. Yang, S. M. Zhang, X. L. Li, and Z. G. Pang, "Variable Sinh-Gaussian solitons in nonlocal nonlinear Schrödinger equation," Applied Mathematics Letters, vol. 82, pp. 64-70, 2018.

[21] J. F. Alzaidy, "The fractional sub-equation method and exact analytical solutions for some nonlinear fractional PDEs," American Journal of Mathematical Analysis, vol. 1, no. 1, pp. 14-19, 2013.

[22] J. F. Alzaidy, "Fractional sub-equation method and its applications to the space-time fractional differential equations in mathematical physics," British Journal of Mathematics and Computer Science, vol. 3, no. 2, pp. 153-163, 2013.

[23] E. M. Zayed, "New traveling wave solutions for higher dimensional nonlinear evolution equations using a generalized $\backslash$ big $\left(\backslash \operatorname{frac}\left\{G^{\wedge}\{\backslash\right.\right.$ prime $\left.\}\right\}\{G\} \backslash$ big) -expansion method," Journal of Physics A, vol. 42, no. 19, p. 195202, 2009.

[24] M. Mirzazadeh, M. Eslami, D. Milovic, and A. Biswas, "Topological solitons of resonant nonlinear Schrödinger's equation with dual-power law nonlinearity by $\left(\mathrm{G}^{0} / \mathrm{G}\right)$-expansion technique," Optik, vol. 125, no. 19, pp. 5480-5489, 2014.

[25] Z. Yan, "Discrete exact solutions of modified Volterra and Volterra lattice equations via the new discrete Sine-Gordon expansion algorithm," Nonlinear Analysis, vol. 64, no. 8, pp. 1798-1811, 2006.

[26] D. Kumar, K. Hosseini, and F. Samadani, "The Sine-Gordon expansion method to look for the traveling wave solutions of the Tzitz'eica type equations in nonlinear optics," Optik, vol. 149, pp. 439-446, 2017.

[27] M. Yavuz, "Novel solution methods for initial boundary value problems of fractional order with conformable differentiation," An International Journal of Optimization and Control: Theories and Applications, vol. 8, no. 1, pp. 1-7, 2018.

[28] M. Yavuz and B. Yaşkıran, "Conformable derivative operator in modelling neuronal dynamics," Applications and Applied Mathematics, vol. 13, no. 2, article 803817, 2018.

[29] M. Yavuz and B. Yaşkıran, "Approximate-analytical solutions of cable equation using conformable fractional operator," New Trends in Mathematical Sciences, vol. 5, no. 4, pp. 209-219, 2017.

[30] M. Yavuz, "Dynamical behaviors of separated homotopy method defined by conformable operator," Konuralp Journal of Mathematics, vol. 7, no. 1, pp. 1-6, 2019.

[31] M. Yavuz and N. Ozdemir, "On the solutions of fractional Cauchy problem featuring conformable derivative," ITM Web of Conferences. EDP Sciences, vol. 22, article 01045, 2018.

[32] C. S. Liu, "The classification of travelling wave solutions and superposition of multi-solutions to Camassa-Holm equation with dispersion," Chinese Physics, vol. 16, no. 7, pp. 1832 1837, 2007.

[33] C. S. Liu, "Representations and classification of traveling wave solutions to Sinh-Gardon equation," Communications in Theoretical Physics, vol. 49, no. 1, pp. 153-158, 2008. 
[34] C. S. Liu, "Solution of ODE $u^{00}+p(u)\left(u^{0}\right)^{2}+q(u)=0$ and applications to classifications of all single travelling wave solutions to some nonlinear mathematical physics equations," Communications in Theoretical Physics, vol. 49, no. 2, pp. 291-296, 2008.

[35] C. S. Liu, “Applications of complete discrimination system for polynomial for classifications of traveling wave solutions to nonlinear differential equations," Computer Physics Communications, vol. 181, no. 2, pp. 317-324, 2010.

[36] H. L. Fan, "The classification of the single traveling wave solutions to the generalized equal width equation," Applied Mathematics and Computation, vol. 219, no. 2, 2012.

[37] Y. Kai, "The classification of the single travelling wave solutions to the variant Boussinesq equations," Pramana, vol. 87, no. 4, p. 59, 2016.

[38] H. L. Fan and L. I. Xin, "The classification of the single travelling wave solutions to the generalized Pochhammer-Chree equation," Pramana, vol. 81, no. 6, p. 925941, 2013.

[39] B. Guan, S. Li, S. Chen, L. Zhang, and C. Wang, "The classification of single traveling wave solutions to coupled timefractional KdV-Drinfel'd-Sokolov-Wilson system," Results in Physics, vol. 13, p. 102291, 2019.

[40] D. Cao, "The classification of the single traveling wave solutions to the time-fraction Gardner equation," Chinese Journal of Physics, vol. 59, pp. 379-392, 2019.

[41] D. Cao, C. Li, and F. He, "Exact solutions to the space-time fraction Whitham-Broer-Kaup equation," Modern Physics Letters B, vol. 34, no. 16, article 2050178, 2020.

[42] C. Shuangqing, L. Yang, W. Lixin, and G. Bing, "Exact solutions to fractional Drinfel'd-Sokolov-Wilson equations," Chinese Journal of Physics, vol. 56, no. 2, pp. 708-720, 2018.

[43] B. Guan, S. Chen, Y. Liu, X. Wang, and J. Zhao, "Wave patterns of $(2+1)$-dimensional nonlinear Heisenberg ferromagnetic spin chains in the semiclassical limit," Results in Physics, vol. 16, p. 102834, 2020.

[44] L. Tang, "Exact solutions to conformable time-fractional Klein-Gordon equation with high-order nonlinearities," Results in Physics, vol. 18, p. 103289, 2020.

[45] Y. Kai, S. Chen, B. Zheng, K. Zhang, N. Yang, and W. Xu, "Qualitative and quantitative analysis of nonlinear dynamics by the complete discrimination system for polynomial method," Chaos, Solitons and Fractals, vol. 141, article 110314, 2020.

[46] Y. Kai, S. Chen, K. Zhang, and Z. Yin, "A study of the shallow water waves with some Boussinesq-type equations," Waves in Random and Complex Media, pp. 1-18, 2021. 